\title{
Corpus Wide Argument Mining-A Working Solution
}

\author{
Liat Ein-Dor, Eyal Shnarch, Lena Dankin, Alon Halfon, Benjamin Sznajder, \\ Ariel Gera, Carlos Alzate, Martin Gleize, Leshem Choshen, Yufang Hou, \\ Yonatan Bilu, Ranit Aharonov, Noam Slonim \\ IBM Research AI \\ \{liate, eyals, lenad, alonhal, benjams, arielge, leshem.choshen, yonatanb, ranita, noams\}@il.ibm.com \\ $\{$ carlos.alzate, martin.gleize, yhou\}@ie.ibm.com
}

\begin{abstract}
One of the main tasks in argument mining is the retrieval of argumentative content pertaining to a given topic. Most previous work addressed this task by retrieving a relatively small number of relevant documents as the initial source for such content. This line of research yielded moderate success, which is of limited use in a real-world system. Furthermore, for such a system to yield a comprehensive set of relevant arguments, over a wide range of topics, it requires leveraging a large and diverse corpus in an appropriate manner. Here we present a first end-to-end high-precision, corpus-wide argument mining system. This is made possible by combining sentence-level queries over an appropriate indexing of a very large corpus of newspaper articles, with an iterative annotation scheme. This scheme addresses the inherent label bias in the data and pinpoints the regions of the sample space whose manual labeling is required to obtain high-precision among top-ranked candidates.
\end{abstract}

\section{Introduction}

Starting with the seminal work of Mochales Palau and Moens (2009), argument mining has mainly focused on the following tasks - identifying argumentative text segments within a given document; labeling these text segments according to the type of argument and its stance; and elucidating the discourse relations among the detected arguments. Typically, the considered documents were argumentative in nature, taken from a well defined domain, such as legal documents or student essays. More recently, some attention had been given to the corresponding retrieval task - given a controversial topic, retrieve arguments with a clear stance towards this topic. This is usually done by first retrieving manually or automatically - documents relevant to the topic, and then using argument mining techniques to identify relevant argumentative segments therein. This documents-based approach was originally explored over Wikipedia (Levy et al. 2014; Rinott et al. 2015), and more recently over the entire Web (Stab et al. 2018). This approach is most suitable for topics of much controversy, where one can find documents directly addressing the debate, in which relevant argumentative text segments are abundant.

Copyright (c) 2020, Association for the Advancement of Artificial Intelligence (www.aaai.org). All rights reserved.
For an argument retrieval system to be of practical use requires: (1) high precision, and (2) wide coverage. The former is important because in order to make a case one typically employs several arguments, all of which need to be relevant and persuasive. For example, if one aims to retrieve 3 such arguments, then even a precision of 0.9 might not be enough, as it implies a 0.27 probability of getting at least one of the arguments wrong. While previous work made great advancements in argument retrieval, such high precision was far from being attained. The second requirement is what sets a practical system from a proof-of-concept. Indeed, previous work mostly focused on a small set of topics and a corresponding moderate-sized corpus. Here we achieve wide coverage by demonstrating how to perform corpus-wide argument mining over massive corpora.

Our starting point is the approach of Levy et al. (2017), which was the first to depart from the document-based dogma, aiming to detect topic-relevant arguments by indexing the sentences of a corpus, and retrieving them directly. This Sentence-Level (SL) approach can potentially detect a much larger set of argumentative texts taken from a wider set of articles, including articles not focused on the topic directly, and which are probably overlooked by the documentlevel approach.

On the other hand, a main challenge emerging from the SL approach is the skewness of the data. When such an approach is applied naively, only a very small fraction of the data are positive examples (relevant arguments). Such an imbalanced dataset is known to be the bane of many supervised machine-learning algorithms. Indeed, this problem is also apparent in the document-level approach, in cases where it is common for the topic to be discussed also in nonargumentative contexts, but is more acute with the SL approach. Previous work either focused on topics for which argumentative content is abundant, or used weak-supervision to try and mitigate the problem.

Our approach is different from previous SL works in three important aspects: (1) We demonstrate for the first time how to leverage supervised-learning techniques for this task, achieving considerably stronger results than earlier works, which adopted the weak-supervision paradigm; (2) We develop an efficient labeling methodology, reminiscent to active learning, which, alongside query-based filtering of the corpus, addresses the data imbalance problem; (3) we report 
results over a massive newspaper corpus, containing close to 10 billion sentences, while earlier SL works reported results only for Wikipedia, which is $\sim 50$ times smaller. We note that the queries used in (2) are similar in essence to the rules used for weak supervision by Levy et al. (2017).

Our approach consists of two steps. First, dedicated queries are applied to retrieve an initial set of potentially relevant sentences. Second, a classifier determines which of the retrieved sentences are both argumentative and relevant. To train such a classifier, one needs to construct a corresponding labeled dataset, which ideally is balanced between the positive and negative examples. However, the fraction of argumentative sentences among those retrieved is typically rather small. To overcome this, we propose an iterative retrospective-labeling approach, of labeling the top predictions of the system, and then training on the obtained labeled data. To bootstrap the process, we start with a modestsized set of labeled data - for example, one obtained via the document-based methods.

The resulting approach is precision-oriented. Clearly, arguments within sentences that do not satisfy the queries we start with, will be missed by such a system. Nonetheless, the experimental results herein suggest that when this approach is coupled with a very large corpus it is able to retrieve, with high precision, a large and diverse set of arguments for a diverse set of topics.

Importantly, any "real world" retrieval system needs to be efficient - it is unfeasible to classify each sentence in the corpus for whether or not it is a relevant argument. Thus, some filtering method must be applied, be it document-retrieval, which limits the scope to the sentences of the retrieved documents, or, as we do here, SL, which limits the scope to sentences matching the queries.

Arguments can be varied and complex. Perhaps the simplest ones, which also serve as building blocks for more complex arguments, are Claims and Evidence (Toulmin 1958). Thus, the complexity of the problem we examine may vary according to the the types of arguments sought; asking for a very specific argument type is a more difficult problem, since these are harder to come by and because the problem is compounded by the need to discern their type. Importantly, in the document-level approach, it is not clear how to aim for documents that are rich with a specific type of arguments. Our SL approach is more flexible in this sense, since by properly designing the queries, one can explicitly search for arguments of a desired type.

To demonstrate the applicability of our approach, we focus on the retrieval of Evidence, specifically those Rinott et al. (2015) denoted as Expert Evidence and Study Evidence. Thus, our system can be perceived as a tool that facilitates critical evaluation of a given topic by providing evidence which support or contest the common claims and beliefs surrounding the topic. In this manner it may be useful to alleviate some of the concerns around fake news. Nonetheless, the methodology presented here is applicable to other argument types as well.

In summary, the main contribution of this work is in presenting a high-precision wide-coverage argument retrieval system, validated over a massive real-world corpus, for a di- verse set of topics. Importantly, this is achieved by a new labeling paradigm that yields a large and balanced annotated dataset over this corpus, and by combining SL queries with a highly effective supervised-learning classifier.

\section{Related Work}

Starting with the work of Mochales Palau and Moens (2009), who looked at legal documents, argument mining has initially focused on documents from specific domains, which tend to be argumentative by nature. In addition to legal documents (Moens et al. 2007; Wyner et al. 2010; Grabmair et al. 2015), examples also include student essays (Stab, Miller, and Gurevych 2018; Persing and $\mathrm{Ng}$ 2016), user comments on proposed regulations (Park and Cardie 2014) and newspaper articles (Feng and Hirst 2011). As with other fields in NLP, deep learning has proven to be a powerful tool for such tasks, be it direct argument mining (Eger, Daxenberger, and Gurevych 2017), predicting argument persuasiveness (Habernal and Gurevych 2016; Gleize et al. 2019), detecting context dependent claims and evidence (Laha and Raykar 2016), or inferring relations among arguments (Cocarascu and Toni 2017; Persing and $\mathrm{Ng}$ 2016). See, for example (Cabrio and Villata 2018; Lawrence, Visser, and Reed 2019) for recent reviews of the field.

Corpus-wide argument mining was originally suggested by Levy et al. (2014). They considered 32 controversial topics, manually identified 326 relevant Wikipedia articles, and labeled some 50000 sentences appearing in these articles for whether or not they contain a claim with a clear stance towards the topic. Similarly, Rinott et al. (2015) aimed to detect evidence for 58 different topics, over 547 manuallycollected articles, and Stab et al. (2018) did so for 8 controversial topics, while considering the top 50 Google-retrieved web pages for each topic.

These methods have achieved moderate success. For example, Levy et al. (2014) report that among their top 50 predictions, on average, precision is 0.19 and recall is 0.4 (a random baseline achieves precision of 0.02). In Stab et al. (2018), where $44 \%$ of the sentences are positive examples, and all sentences are classified, the best F1-score is 0.66 (an "all-yes" baseline has an F1-score of 0.61).

We envision a "real world" argument retrieval system as one which retrieves several arguments, that are typically all relevant to the topic. Hence, such a system requires high precision, well beyond the aforementioned accuracy-oriented systems. Moreover, it should work well on a wide variety of topics, many of which are probably not associated with such argument-abundant articles as in the data of Stab et al. (2018). Both of these goals are achieved in this work.

Other work on argument retrieval includes Wachsmuth et al. (2017) and Al-Khatib et al. (2016), who also consider cross-topic argument mining, but do so over a dataset of arguments rather than a heterogeneous corpus where only a small fraction of the text is argumentative. Similar to our work here, Shnarch et al. (2018) also aim to classify sentence candidates for whether or not they are evidence, but their emphasis is on a semi-supervised method, applied to Wikipedia, without addressing the sentence-retrieval task. 
The iterative retrospective labelling technique suggested here may seem similar to the semi-supervised approach of Label Propagation (Zhu and Ghahramani 2002). In Label Propagation, unlabeled data is pseudo-labeled automatically according to the labels of similar data, and similarity is computed automatically. By contrast, we do not attempt to define similarity between arguments, and unlabeled data of interest is manually labeled.

Another related labelling approach is Active Learning (Cohn, Ghahramanilahar, and Jordan 1996), an umbrella term covering a variety of algorithms which aim to select informative instances for labelling, in order to learn a suitable classifier. Previous studies have indicated that the performance of active learning is easily disrupted by an imbalanced data distribution (Zhu and Hovy 2007; Bloodgood and Vijay-Shanker 2009). Moreover, in most active learning literature, accuracy is chosen as the evaluation metric, while in our case, the quantity of interest is precision. A recent work (Wang et al. 2018) suggested a method for optimizing expected average precision in active learning, but did not deal with the problem of skewed class distribution. To the best of our knowledge, our iterative retrospective labelling technique is the first precision-oriented active learning strategy for coping with the class-imbalance problem. Moreover, while Wang et al. (2018) are interested in average precision, which is affected by the entire set of examples, in our task it is the top-ranked predictions which are of interest.

\section{Definitions}

Following the nomenclature of competitive debate and previous papers (e.g. Levy et al. 2014), we define a Motion as a high-level claim implying some clearly positive or negative stance towards a (debate's) topic, and, optionally some policy or action that should be taken as a result. For example, a motion can be We should ban the sale of violent video games or Capitalism brings more harm than good. In the first case, the topic is violent video games, and the proposed action is ban. In the second case, the topic is Capitalism, and no action is proposed. In this work, the topic of motions will always be a reference to a Wikipedia article.

In the context of a motion, we define Evidence as a single sentence that clearly supports or contests the motion, yet is not merely a belief or a claim. Rather, it provides an indication whether a belief or a claim is true.

Specifically, we are interested in two types of Evidence: Study Evidence, which presents a quantitative analysis of data, and Expert Evidence which presents testimony by a relevant expert or authority (Rinott et al. 2015).

For example, the sentence The research clearly suggests that, among other risk factors, exposure to violent video games can lead to aggression and other potentially harmful effects is Study Evidence supporting the motion, and specifically supports the claim that violent video games can lead to aggression.

\section{Data Acquisition}

In supervised learning, class imbalance in training data distribution often causes learning algorithms to perform poorly on the minority class. This issue has been well addressed in the machine learning literature (Chawla, Japkowicz, and Kotcz 2004; Garcia, Mollineda, and Sanchez 2010; Van Hulse, Khoshgoftaar, and Napolitano 2007). The corpus we consider in this work is very large, containing some 400 million articles, in which, for nearly any motion, positive examples are few and far between. This poses the question of how to obtain training data which is relatively balanced, while not wasting much of the labeling effort on sentences which would later be discarded.

Our approach is as follows. Start with a small set of manually collected and labeled sentences - which can be obtained by manually querying the corpus, or by taking data from a smaller corpus, which is enriched with positive examples. Next, train a simple classifier, such as logistic regression, which can generalize well based on this dataset, and use it to predict the label of many relevant sentences in the very large corpus (e.g., sentences that satisfy specific queries). Labeling the top predictions of the classifier, one obtains a larger dataset. Note that in this second phase the set of motions can be expanded well beyond the original one, further increasing the size of the dataset.

Labeling the top predictions has two advantages. First, since they are enriched with true-positive examples, this helps lessen the label imbalance in the dataset. Second, it brings forth the "hard" negative examples; those which are likely to be the main obstacle for obtaining what we seek for a "real world" retrieval system: a high precision among the top-ranked candidates. By labeling these "hard" examples the classifier is improved in the relevant regions of the sample space.

Once the dataset is large enough, and not too skewed, one can train a more powerful classifier, such as a neural net. The dataset and classifier can then be improved in a similar manner, by iteratively labeling the top predictions of the new classifier, and presumably increasing the fraction of positive examples in each iteration.

Specifically, our starting point is a corpus of some 400 million newspaper and journal articles provided by LexisNexis (which we denote as VLC - Very Large Corpus), and the logistic-regression classifier constructed by Rinott et al. (2015), which is based on a relatively small set of manually labeled Wikipedia sentences. We selected 192 and 47 motions for our train and development sets respectively, and for each motion retrieved sentences in the corpus using queries similar to those in Levy et al. (2017) (see Section 5). The retrieved sentences were then ranked using the logistic-regression classifier, and the top 40 sentences for expert and study evidence were manually annotated using crowd-sourcing ${ }^{1}$. Annotation was binary - whether or not the sentence is an Evidence of the desired type - and the gold label was determined by majority.

The network of Shnarch et al. (2018) was then trained on this dataset, and again the top 40 predictions for each motion and each evidence type were manually annotated. This was iterated until we ended up with a dataset of 198, 457 manually labeled sentences, of which $33.5 \%$ are positive ex-

\footnotetext{
${ }^{1}$ Using the Figure Eight platform, www.figure-eight.com.
} 
amples. We denote this dataset as the Very Large Dataset (VLD).

During the labeling process, each sentence-motion pair was annotated by 10 Figure-Eight annotators. Interannotator agreement (over multiple tasks) was computed via Cohen's Kappa for all pairs of annotators ${ }^{2}$. Then, for each annotator, the average agreement with other annotators - weighted by the number of common items - was computed. Annotators with low average agreement (Cohen's Kappa $<0.3$ ) were discarded. If the number of labels for a sentence-motion pair was below 7, we kept labeling to reach 7 trusted annotations. This yielded an overall average Cohen's Kappa of 0.47 - computed over all pairs of remaining annotators, and weighted by the number of common items. Labels of sentence-motion pairs were determined by majority; ties were taken as negative labels.

Note that a Cohen's Kappa of 0.47 is on par with many previous reports, focusing on type-dependent argumentation datasets (Stab et al. 2018; Stab and Gurevych 2017; Boltužić and Šnajder 2014), reflecting the challenging nature of the task compared to type-independent annotation (Levy et al. 2014; Rinott et al. 2015; Shnarch et al. 2018). Finally, as indicated by the very high precision of the resulting models, the dataset evidently provides a clear signal for evidence detection.

In addition to the dataset above, a matching dataset over Wikipedia was constructed. For the same set of training and development motions as in the VLD, the queries and baseline network described in section 5 were used to retrieve and rank Wikipedia sentences. The top 20 predictions for each motion were then manually annotated. This yielded 29, 429 labeled sentences (of which $23 \%$ are positive examples), which we denote as the Wikipedia Dataset (Wiki) and is available at http://ibm.biz/debater-datasets.

\section{System Architecture}

Sentence retrieval is done using queries that specify which terms should appear and in what order, with some gaps allowed. These terms include the topic, the action, named entities such as numbers, persons and organizations, lexicon terms indicating sentiment, or of particular relevance to the type of evidence sought - Expert or Study, and certain connectors which are indicative of evidence. Importantly, all queries require the topic to appear in the sentence, and so retrieved sentences always contain a corresponding term.

For example, a query oriented towards retrieving Evidence of type Study is defined by requiring that the following elements appear in a sentence in order (but not necessarily adjacent; other words may appear in between): (1) a term from a study related lexicon; (2) the conjunction term That (Levy et al. 2018); (3) the topic; (4) a term from a sentiment lexicon. Accordingly, for the topic gambling, one of the sentences retrieved by the query is: The University of Glasgow and Healthy Stadia research warns that gambling is a public health issue with potential for harm, where the words in bold are those that match the required terms (in order).

\footnotetext{
${ }^{2}$ Whenever agreement was computed, only annotators with at least 50 items in common were considered.
}

A full description of the queries and their associated lexicons is available along with the Wikipedia Dataset files.

Queries are arranged in a cascade, and a total of up to 12,000 sentences is sought for each evidence type. Queries that are more likely to yield evidence are run first (see Supplementary Materials), and the cascade halts once the 12, 000 limit has been reached.

Allowing such queries required indexing the VLC not only for word strings, but also for their relevant semantic roles, such as being a named entity or appearing in one of the lexicons. Moreover, since in our framework the topic is a Wikipedia title by definition (see section 3 ), all sentences are also "wikified", that is, terms are linked to their underlying Wikipedia titles when possible. To allow processing billions of sentences in a timely manner, we use a simple rule-based Wikification method which mainly relies on Wikipedia redirects (Shnayderman et al. 2019).

The output of the retrieval stage is the union of sentences from both the Expert Evidence queries and the Study Evidence queries, with duplicate sentences removed. Figure 1 summarizes the suggested pipeline for evidence retrieval.

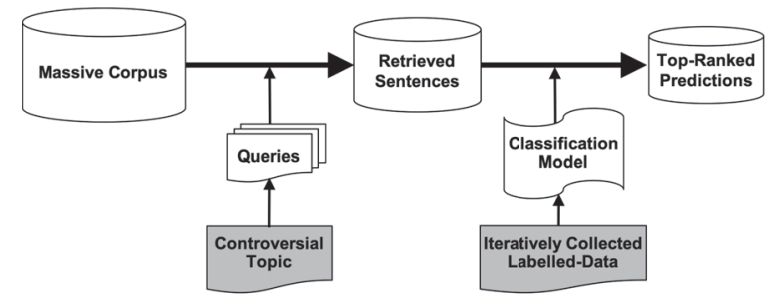

Figure 1: Overview of the end-to-end evidence retrieval system.

Whereas recent work on evidence retrieval (Stab et al. 2018; Shnarch et al. 2018) was limited to considering a small set of documents and correspondingly focused on the classification task, here we describe a full end-to-end retrieval system, and hence focus on ranking the retrieved sentences. Specifically, retrieved sentences are fed to a classifier which computes a score indicating the confidence that the sentence is an evidence to the motion, and ranked according to this score.

\section{Experiments}

To evaluate the suggested end-to-end framework we trained several neural net variants on the VLD, and assessed their performance on four benchmarks.

The network variants differ in the input they receive and in the underlying architecture. We consider the following input variants:

- $\mathrm{S}+\mathrm{M}$ - sentence and motion

- MaskS+M - masked sentence and motion

- MaskS - masked sentence

Masking is done by replacing the topic in a sentence with a special token (recall that all retrieved sentences contain the topic). The purpose of masking is threefold: (1) it marks the 
topic within the sentence; (2) it introduces a uniform representation for the different forms of the same topic; (3) it introduces a uniform representation for the different possible topics.

Masking was also used by Shnarch et al. (2018), but they did not consider adding the motion as input. Hence, they in fact framed the task as deciding whether or not a sentence is evidence for some implicit motion. By making the motion explicit, the network's training is ostensibly more aligned with what it is actually meant to do - determine whether or not a sentence is evidence for a given motion.

We examine two families of network architectures:

- BA - the network described in Shnarch et al. (2018). A bidirectional LSTM (Graves and Schmidhuber 2005) with an additional self attention layer (Yang et al. 2016), followed by a fully connected layer.

- BERT - a network based on BERT (Devlin et al. 2018), where the pre-trained model is fine-tuned using our labeled dataset.

The motivation for choosing BA for our experiments is that it was successfully used by Shnarch et al. (2018) for sentence classification in the context of evidence detection. We aimed to determine the effect of training this network on the much larger dataset that we were able to collect with the methods described above, and to see how the different input variants affect its performance. We chose BERT since it presents state-of-the-art results in a wide variety of NLP tasks. Notice that $\mathrm{BA}$ receives a single text as input. In order to train a BA-based network which also receives the motion as input (i.e., the $\mathrm{S}+\mathrm{M}$ and $\mathrm{MaskS+M}$ variants), we made the following modifications to the embedding layer of BA. We embed both the sentence and the topic using the same BA embedding layer (BiLSTM+attention). We then obtain the joint embedding by concatenating the subtraction and the pointwise product of the two resultant embeddings, and add a fully connected layer on top of the joint embedding to obtain the output. All BA-based models were trained with the cross-entropy loss function.

The four benchmarks used for evaluation are as follows. The first and second benchmarks were obtained by running an end-to-end system on large corpora - one being the VLC, and the other Wikipedia (2018 dump) - for 100 novel motions. Top predictions were then manually annotated to directly assess ranking, and precision was computed as a function of the number of predictions made (annotation is done in the same way as for the training data, and over the top predictions of all network variants). For completeness, we also trained all networks on the Wiki dataset, and assessed their end-to-end precision over the Wikipedia benchmark.

Importantly, the aim of the evaluation is to simulate the end-to-end performance on unseen motions, focusing on the precision of top predictions. Hence, using a fixed test data is problematic, since it does not reflect the predictions of the considered model, but a somewhat arbitrary set accumulated during the iterative labeled data collection.

Note that results are comparable only when considering the same benchmark, but not between benchmarks. The VLC, containing some 100 times more documents, is ex- pected to have far more relevant evidence than Wikipedia. So, for example, when computing the precision over the top $k$ predictions, Wikipedia might simply not contain $k$ relevant sentences, and a precision of, say, 1.0 would not be attainable. However, for a given benchmark, since all networks rank the same set of sentences, precision results are comparable. Note also that since the VLC is so big, it is not uncommon to retrieve paraphrases of essentially the same evidence - much more so than in Wikipedia. To mitigate this problem, when ranking VLC sentences, those with high similarity ${ }^{3}$ to higher-ranking ones were removed. This filtering resulted in the removal of $10 \%$ of the retrieved sentences.

The third and fourth benchmarks compare our system to previous works - Shnarch et al. (2018) and Stab et al. (2018) - in which the sentences are given, and the goal is to classify them. We denote these benchmarks as BlendNet and UKP$T U D A$, respectively. We evaluate our networks on the benchmarks in the same way as in the original works - computing accuracy on BlendNet, and precision-recall on UKP-TUDA. To do this, networks' scores are converted to binary labels by taking a threshold of 0.5 (all variants were trained with loss functions w.r.t. binary labels).

Since the original works did not filter out similar sentences, we did not do so for these benchmarks. Additionally, as some of the motions in the test set of Shnarch et al. (2018) also appear in the VLD training and dev sets, models were retrained for the purpose of evaluation on this benchmark, by excluding motion-sentence pairs corresponding to overlapping motions from the train and development sets.

\section{Results}

\subsection{End-to-end System}

We evaluate the end-to-end performance of the different models using two benchmarks, VLD and Wiki on the 100 test motions.

VLD Figure 2 presents the average precision of different evidence detection models as a function of the number of top candidates per motion. Precision is high for all models, with over $90 \%$ precision for the top 20 candidates, and a remarkably high precision of $95 \%$ for the best model over the top 40 candidates. This compares to an estimated positive prior of 0.3 among the sentences retrieved from the queries ${ }^{4}$. The best performing model is BERT $_{S+M}$, with precision values that clearly surpass those obtained by the $\mathrm{BA}_{M a s k S}$ model used by Shnarch et al. (2018). Notice that the $\mathrm{BA}_{M a s k S}$ model receives only the masked sentence as input, while BERT $_{S+M}$ receives the unmasked sentence and the motion. As expected, both BA and BERT benefit from the addition of the motion text to the input (see Figure 2).

Among the BA based models, the best results are achieved when the input is the masked sentence and the motion text $\left(\mathrm{BA}_{M a s k S+M}\right)$. As mentioned in Section 6, the advantage

\footnotetext{
${ }^{3}$ Word overlap of at least 0.8 w.r.t. the shorter sentence; excluding stop-words and the topic.

${ }^{4}$ The estimate is based on labelling 10 random sentences for each of the 100 test motions.
} 
of masking can be attributed to the extra information it provides to the network. First, it conveys to the network where the topic is located within the sentence. Second, the topic may appear in multiple textual forms. For example, the topic "capital punishment" may appear in the text as "execution", "death penalty", etc. Replacing Wikified instances of the topic with a single mask saves the need to learn the equivalence between the different forms. Finally, the usage of the same masking token for all topics can help the network learn general, motion-independent features of evidence texts.

In the case of BERT, the masking hinders the network performance. Models that are fed with masked sentences are inferior to $\mathrm{BERT}_{S+M}$, which receives an unmasked sentence and a motion. A possible explanation for the negative effect of masking on BERT is that its strong underlying language model enables it to deduce the aforementioned information provided by masking from the original unmasked text. Consequently, the information that is lost in the masking process, i.e. the masked tokens, leads to inferior results.

It is also interesting to evaluate to what extent the direct sentence-retrieval approach differs from one based on document-retrieval. In other words, do high-ranking sentences come from a wide range of documents and journals, or is this approach de-facto similar to the document-based one, with high-ranking sentences originating from only a small number of documents? As shown in Figure 3, the former is true. For example, the top 20 and top 40 ranked candidates per motion from the $\mathrm{BA}_{\text {MaskS }}$ model originate from an average of 18.03 and 36.07 different documents, respectively.

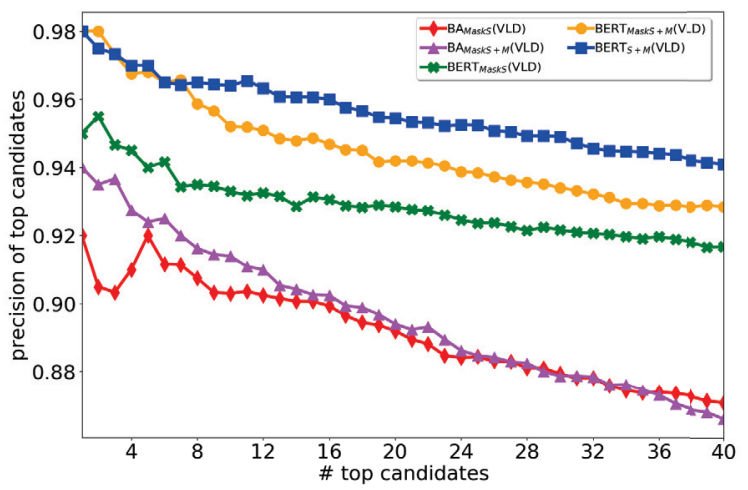

Figure 2: VLD: Precision of top $k$ candidates vs. $k$ for different evidence detection models.

Wiki Following the high performance of the end-to-end system on VLD, it is interesting to examine how the results are affected by moving to the smaller and more homogeneous corpus of Wiki. For this purpose we tested the models trained using VLD, on Wiki test-set. In addition we trained new models on Wiki training-set and tested them on the Wiki test-set. Figure 4 shows the average precision of our models vs. the number of top candidates. Although the precision values of the leading model are still high, they are

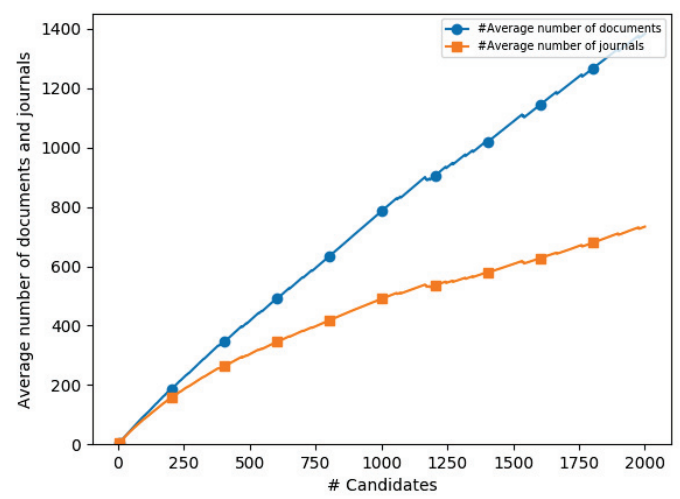

Figure 3: Average number of documents and journals from which the top $k$ candidates originate, as a function of $k$. Results are based on top candidates of BERT $_{S+M}$ (VLD).

significantly lower than those obtained in VLD. One possible reason is the difference in domains, that is, that the distributions of sentences retrieved from VLD and those retrieved from Wiki are somewhat different. Another reason may be that the dramatically smaller number of sentences in Wiki is likely to result in lower scores among the top ranked candidates ${ }^{5}$. The lower the score the less likely it is that a sentence is evidence. Indeed, the Wiki scores over the top $\mathrm{k}$ predictions are significantly lower than the VLD scores ( $\mathrm{t}$-test $\mathrm{p}$-value $=3.19 e^{-9}$ for $\left.k=20\right)$. Despite the performance differences when moving from VLD to Wiki, the hierarchy between the different models is the same. Moreover, although the test sentences are from Wiki, the models which are trained on Wiki are inferior to those trained on VLD. This can be attributed to the much larger training data of VLD (154K compared to $22 \mathrm{~K}$ in Wiki), which compensates for the potential difference between the train and test distributions.

\subsection{BlendNet Benchmark}

The $\mathrm{BA}_{\text {MaskS }}$ network used here has the same architecture as that in Shnarch et al. (2018). It is therefore interesting to evaluate how the differences between their work and ours - greatly expanding the training data, considering the motion explicitly and using the BERT architecture - are reflected over the benchmark based on their data. Table 1 describes the impact of these differences. Training $\mathrm{BA}_{M a s k S}$ on the VLD improves accuracy from $74 \%$ to $78 \%$. In other words, even though BlendNet was trained on Wikipedia sentences, and the benchmark is also composed of Wikipedia sentences, the larger size of the VLD more than compensates for the change in domain. Shifting to the BERT architecture improves accuracy to $81 \%$, even without adding the motion as part of the input. Finally, the best accuracy is at-

${ }^{5}$ Let $X$ be the number of sentences; the top $k$ candidates correspond to the top $k / X$ percentile of scores. For $Y<X$, the percentile of the top $k$ candidates becomes $k / Y>k / X$, which corresponds to lower scores. 


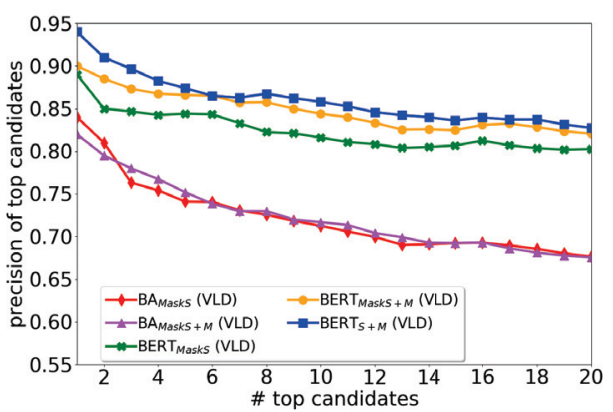

(a) Trained on VLD

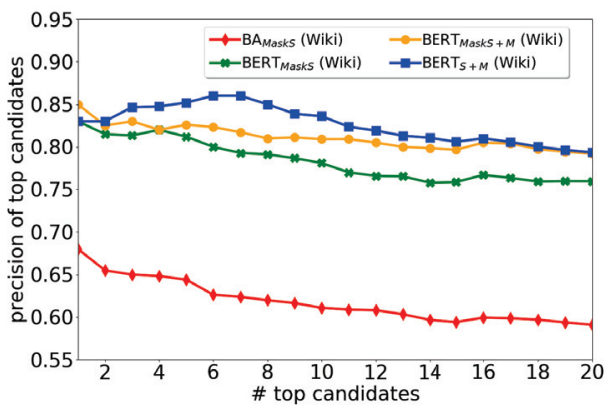

(b) Trained on Wiki

Figure 4: Wiki: Precision of top $k$ candidates vs. $k$ for different evidence detection models.

\begin{tabular}{|c|c|c|}
\hline Arch. & trained on & Accuracy \\
\hline BA $_{M a s k S}$ & (Shnarch et al. 2018) & 0.74 \\
\hline $\mathrm{BA}_{M a s k S}$ & VLD & 0.78 \\
\hline $\mathrm{BA}_{\text {Mask }+M}$ & VLD & 0.77 \\
\hline BERT $_{M a s k S}$ & VLD & 0.81 \\
\hline BERT $_{M a s k S+M}$ & VLD & 0.82 \\
\hline BERT $_{S+M}$ & VLD & 0.84 \\
\hline
\end{tabular}

Table 1: Accuracy of sentence classification over BlendNet.

tained when combining all three modification, for a total of nearly $14 \%$ improvement over the best results reported by Shnarch et al. (2018).

\subsection{UKP-TUDA Benchmark}

The UKP-TUDA benchmark is an interesting case study for our networks, since it is labeled for whether or not a sentence is argumentative (defined as having a clear stance w.r.t. the topic), rather than whether or not it is evidence. In particular, argumentative texts which are not Study Evidence or Expert Evidence are labeled as positive examples, but are expected to receive a low score by $\mathrm{BERT}_{S+M}$. In other words, when naively used as a classifier, BERT $_{S+M}$ is expected to accept argumentative sentences which are evidence of the appropriate type, but reject argumentative sentences which are not.

Indeed, a threshold of 0.5 leads to a precision score of 0.88 and a recall score of 0.16 . To verify that the predicted positives indeed tend to be evidence and that the low recall is due to other types of argumentative sentences being rejected, we manually annotated 20 argumentative sentences with a score above the threshold, and 20 with a score below it (chosen uniformly at random). As expected, in the first set, 14 of the sentences are Study Evidence or Expert Evidence, while in the second set only 2 are so.

Importantly, BERT $S+M$ was trained on sentences which correspond to the retrieval queries, and are therefore quite different from the sentences in the UKP-TUDA benchmark; In particular, it is not clear what fraction of the negative training examples are non-argumentative sentences. Hence, it is somewhat unclear how such sentences will be scored. To evaluate this, we considered all sentences with a classification score below the threshold. Among those which are argumentative, the average score is $7.3 \cdot 10^{-2}$, while among those which are not argumentative it is $1.5 \cdot 10^{-2}$ (t-test $\mathrm{p}$-value $<10^{-76}$ ), suggesting that argumentative sentences tend to be assigned a higher score.

A different way to examine this phenomenon is by considering the precision-recall curve over this benchmark. Figure 5 shows that even though the network was trained to discern evidence sentences, it also learned to prefer argumentative sentences over non-argumentative ones. The curve-points toward its right end correspond to precision-recall trade-offs similar to those reported in Stab et al. (2018) for classifiers trained directly for this task. For example, a threshold score of 0.002 corresponds to precision of 0.66 and recall of 0.75 $(\mathrm{F} 1$-score $=0.7)$ on this curve. The preferred network in Stab et al. (2018) achieves an average precision of 0.65, 0.67 average recall and 0.67 average F1-score (see Figure 5).

Taken together these results suggest that even though BERT $_{S+M}$ was trained on rather different data, its ranking of the UKP-TUDA tends to be Study Evidence or Expert Evidence first, then argumentative sentences not of these types and last but not least - the non-argumentative sentences.

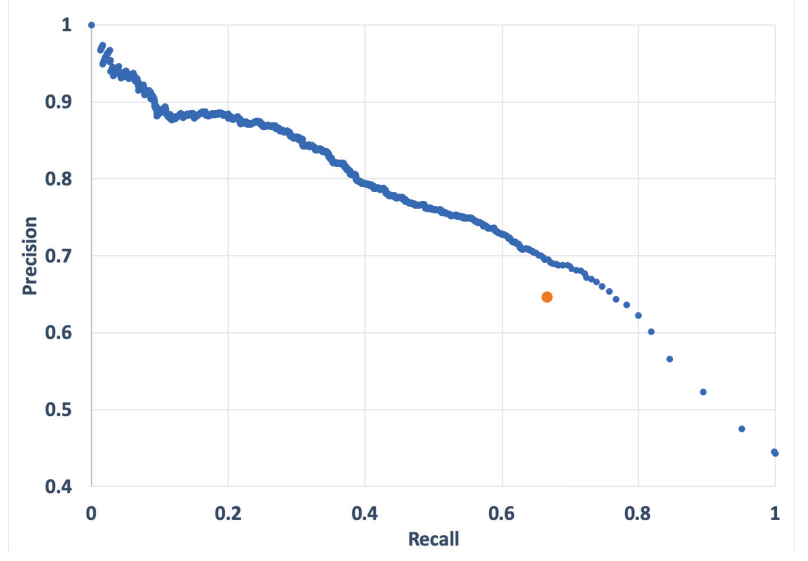

Figure 5: Precision-Recall curve of BERT $_{S+M}$ over the UKP-TUDA benchmark. The orange mark denotes the best result reported by Stab et al. (2018).

Finally, to appreciate that the task of retrieving relevant evidence can be quite nuanced and challenging, the table in 


\begin{tabular}{|c|c|c|}
\hline Motion & Positive example & Negative example \\
\hline $\begin{array}{l}\text { Blood } \\
\text { donation } \\
\text { should be } \\
\text { mandatory }\end{array}$ & $\begin{array}{l}\text { A study published in the American Journal of } \\
\text { Epidemiology found that blood donors have } \\
\text { 88-percent less risk of suffering from a heart } \\
\text { attack and stroke. }\end{array}$ & $\begin{array}{l}\text { Statistics from the Nakasero Blood Bank show } \\
\text { that students are the main blood donors } \\
\text { contributing about } 80 \text { per cent of the blood } \\
\text { collected countrywide. }\end{array}$ \\
\hline $\begin{array}{l}\text { Child labor } \\
\text { should be } \\
\text { legalized }\end{array}$ & $\begin{array}{l}\text { FAO stressed that child labor in agriculture is a } \\
\text { global problem that harms children, harms the } \\
\text { agricultural sector and perpetuates rural poverty. }\end{array}$ & $\begin{array}{l}\text { FAO supports governments to ensure that child } \\
\text { labour issues are better integrated into national } \\
\text { agriculture development policies and strategies. }\end{array}$ \\
\hline $\begin{array}{l}\text { Force- } \\
\text { feeding } \\
\text { should be } \\
\text { banned }\end{array}$ & $\begin{array}{l}\text { The IMA argued against the amendment on the } \\
\text { grounds that force-feeding can pose a serious } \\
\text { danger to the prisoner's health and violates the } \\
\text { ethical rule of doing no harm. }\end{array}$ & $\begin{array}{l}\text { In Washington, Senate leaders continue efforts to } \\
\text { force-feed an unpopular Obamacare repeal that } \\
\text { will eliminate health coverage for } 1.3 \text { million } \\
\text { North Carolinians who are now covered. }\end{array}$ \\
\hline $\begin{array}{l}\text { We should } \\
\text { abandon } \\
\text { Valentine's } \\
\text { day }\end{array}$ & $\begin{array}{l}\text { The Canadian polling firm Insights West surveyed } \\
\text { a representative sample of Canadians who are in a } \\
\text { relationship and found that } 62 \text { percent agreed that } \\
\text { Valentine's Day is a waste of time and money. }\end{array}$ & $\begin{array}{l}\text { A recent survey by Virgin Mobile USA found that } \\
59 \text { percent of people said that if they were going } \\
\text { to break up with someone, they would do so just } \\
\text { before Valentine's Day to save money. }\end{array}$ \\
\hline
\end{tabular}

Figure 6: Example of sentences containing similar terms, of which only one is a relevant evidence. Similar terms are in the same color.

Figure 6 lists several motions, each with a pair of retrieved sentences. In each case, the pair of sentences contain similar terms, but nonetheless one is a relevant evidence, and the other is not. All these examples were successfully discerned by the suggested model.

\section{Discussion and Conclusion}

We presented a first end-to-end "working solution" for the argument retrieval task, showing that it attains remarkably high precision on a wide range of topics. Moreover, in spite of being trained on data of a somewhat different nature, our best model attained results comparable to previous state-ofthe-art in one benchmark data (Stab et al. 2018), and significantly outperformed state-of-the-art on a second benchmark data (Shnarch et al. 2018). A key element of our approach is the Retrospective Labeling paradigm, a precision-oriented Active Learning, which is targeted to cope with skewed label distribution. This strategy is of general applicability, especially in retrieval tasks where precision is the common evaluation metric and positive examples are scarce.

We have suggested the SL approach as an alternative to the document-based one, but it can actually complement it. One can envision a hybrid solution that may enjoy the best of both worlds. One approach could be to simply combine sentences retrieved by both methods. Alternatively, one could start with SL retrieval, and then leverage the SL predictions to identify relevant documents or passages. Specifically, one could run the SL based system suggested here, and then expand each of the top-ranking sentences into the containing paragraph or document. Presumably, such a method would enjoy both the wide variety of contexts in which SL-based arguments are found, while alleviating the restrictions that retrieved sentences conform to one of the SL-queries.

Another concession that was made here in the interest of precision is limiting the scope of retrieval to sentences conforming to the queries' patterns. It is interesting to try and expand this, in particular to sentences which do not mention the topic explicitly. One approach could be to try and solve the co-reference problem during indexing, thus being able to retrieve sentences in which the topic is only referenced. Another approach would be to first expand the topic, and then do argument-retrieval for the expanded set of topics (Bar-Haim et al. 2019).

Our work focused on the retrieval of specific types of Evidence, but the same approach can be applied to other types of arguments. In particular, we used the same approach to retrieve Claims with high precision; the details of these analyses are omitted due to lack of space.

Moreover, argument-retrieval can be defined at different levels of granularity - e.g., retrieving arguments of any type, retrieving only Evidence, retrieving only Study Evidence, or retrieving only certain types of Study Evidence such as polls. Arguments of different types share many commonalities due to their argumentative nature, as shown by employing the model developed here on the data of Stab et al. (2018). Accordingly, Transfer Learning techniques can probably exploit the large annotated dataset herein for the task of retrieving arguments of other types.

Similarly, it is interesting to explore how Domain Adaptation techniques can leverage labels from an annotated corpus such as those presented here to novel ones. Namely, while we have shown that obtaining high-quality, balanced labeled data from a massive corpus is plausible, it does nonetheless require a considerable annotation effort. Hopefully, when presented with a corpus from a new domain, the models developed here can be adapted to effectively retrieve arguments from that corpus as well.

\section{References}

Al-Khatib, K.; Wachsmuth, H.; Hagen, M.; Köhler, J.; and Stein, B. 2016. Cross-domain mining of argumentative text through distant supervision. In NAACL 2016, 1395-1404.

Bar-Haim, R.; Krieger, D.; Toledo-Ronen, O.; Edelstein, L.; Bilu, Y.; Halfon, A.; Katz, Y.; Menczel, A.; Aharonov, R.; and Slonim, N. 2019. From surrogacy to adoption; from bitcoin to cryptocurrency: Debate topic expansion. In ACL 2019, 977-990. 
Bloodgood, M., and Vijay-Shanker, K. 2009. Taking into account the differences between actively and passively acquired data: The case of active learning with support vector machines for imbalanced datasets. In NAACL, 137-140.

Boltužić, F., and Šnajder, J. 2014. Back up your stance: Recognizing arguments in online discussions. In Proceedings of the First Workshop on Argumentation Mining, 49-58. Baltimore, Maryland: Association for Computational Linguistics.

Cabrio, E., and Villata, S. 2018. Five years of argument mining: a data-driven analysis. In IJCAI, 5427-5433.

Chawla, N. V.; Japkowicz, N.; and Kotcz, A. 2004. Special issue on learning from imbalanced data sets. ACM Sigkdd Explorations Newsletter 6(1):1-6.

Cocarascu, O., and Toni, F. 2017. Identifying attack and support argumentative relations using deep learning. In EMNLP 2017, 1374 1379.

Cohn, D. A.; Ghahramanilahar, Z.; and Jordan, M. I. 1996. Active learning with statistical models. Journal of artificial intelligence research 4:129-145.

Devlin, J.; Chang, M.-W.; Lee, K.; and Toutanova, K. 2018. Bert: Pre-training of deep bidirectional transformers for language understanding. arXiv preprint arXiv:1810.04805.

Eger, S.; Daxenberger, J.; and Gurevych, I. 2017. Neural endto-end learning for computational argumentation mining. In $A C L$ 2017, 11-22.

Feng, V. W., and Hirst, G. 2011. Classifying arguments by scheme. In ACL 2011, 987-996.

Garcia, V.; Mollineda, R. A.; and Sanchez, J. S. 2010. Theoretical analysis of a performance measure for imbalanced data. In $I C P R$ 2010, 617-620.

Gleize, M.; Shnarch, E.; Choshen, L.; Dankin, L.; Moshkowich, G.; Aharonov, R.; and Slonim, N. 2019. Are you convinced? choosing the more convincing evidence with a siamese network. In $A C L$ 2019, 967-976.

Grabmair, M.; Ashley, K. D.; Chen, R.; Sureshkumar, P.; Wang, C.; Nyberg, E.; and Walker, V. R. 2015. Introducing luima: An experiment in legal conceptual retrieval of vaccine injury decisions using a uima type system and tools. In ICAIL'15, 69-78.

Graves, A., and Schmidhuber, J. 2005. Framewise phoneme classification with bidirectional lstm and other neural network architectures. Neural Networks 18(5-6):602-610.

Habernal, I., and Gurevych, I. 2016. Which argument is more convincing? analyzing and predicting convincingness of web arguments using bidirectional lstm. In ACL 2016, 1589-1599.

Laha, A., and Raykar, V. C. 2016. An empirical evaluation of various deep learning architectures for bi-sequence classification tasks. CoRR abs/1607.04853.

Lawrence, J.; Visser, J.; and Reed, C. 2019. An online annotation assistant for argument schemes. In Proceedings of the 13th Linguistic Annotation Workshop, 100-107.

Levy, R.; Bilu, Y.; Hershcovich, D.; Aharoni, E.; and Slonim, N. 2014. Context dependent claim detection. In COLING, 1489-1500.

Levy, R.; Gretz, S.; Sznajder, B.; Hummel, S.; Aharonov, R.; and Slonim, N. 2017. Unsupervised corpus-wide claim detection. In Argument Mining Workshop, 79-84.

Levy, R.; Bogin, B.; Gretz, S.; Aharonov, R.; and Slonim, N. 2018. Towards an argumentative content search engine using weak supervision. In COLING 2018, 2066-2081.
Mochales Palau, R., and Moens, M.-F. 2009. Argumentation mining: the detection, classification and structure of arguments in text. In ICAIL 2009, 98-109.

Moens, M.-F.; Boiy, E.; Palau, R. M.; and Reed, C. 2007. Automatic detection of arguments in legal texts. In ICAIL '07, 225-230.

Park, J., and Cardie, C. 2014. Identifying appropriate support for propositions in online user comments. In Arg Mining Workshop, 29-38.

Persing, I., and Ng, V. 2016. End-to-end argumentation mining in student essays. In NAACL 2016, 1384-1394.

Rinott, R.; Dankin, L.; Alzate Perez, C.; Khapra, M. M.; Aharoni, E.; and Slonim, N. 2015. Show me your evidence - an automatic method for context dependent evidence detection. In EMNLP 2015, $440-450$.

Shnarch, E.; Alzate, C.; Dankin, L.; Gleize, M.; Hou, Y.; Choshen, L.; Aharonov, R.; and Slonim, N. 2018. Will it blend? blending weak and strong labeled data in a neural network for argumentation mining. In ACL 2018, 599-605.

Shnayderman, I.; Ein-Dor, L.; Mass, Y.; Halfon, A.; Sznajder, B.; Spector, A.; Katz, Y.; Sheinwald, D.; Aharonov, R.; and Slonim, N. 2019. Fast end-to-end wikification. arXiv.

Stab, C., and Gurevych, I. 2017. Parsing argumentation structures in persuasive essays. Computational Linguistics 43(3):619-659.

Stab, C.; Miller, T.; Schiller, B.; Rai, P.; and Gurevych, I. 2018. Cross-topic argument mining from heterogeneous sources. In EMNLP 2018, 3664-3674.

Stab, C.; Miller, T.; and Gurevych, I. 2018. Cross-topic argument mining from heterogeneous sources using attention-based neural networks. CoRR abs/1802.05758.

Toulmin, S. E. 1958. The Uses of Argument. Cambridge University Press.

Van Hulse, J.; Khoshgoftaar, T. M.; and Napolitano, A. 2007. Experimental perspectives on learning from imbalanced data. In ICML 2007, 935-942. ACM.

Wachsmuth, H.; Potthast, M.; Al Khatib, K.; Ajjour, Y.; Puschmann, J.; Qu, J.; Dorsch, J.; Morari, V.; Bevendorff, J.; and Stein, B. 2017. Building an argument search engine for the web. In Argument Mining Workshop, 49-59.

Wang, H.; Chang, X.; Shi, L.; Yang, Y.; and Shen, Y.-D. 2018. Uncertainty sampling for action recognition via maximizing expected average precision. In IJCAI 2018, 964-970.

Wyner, A.; Mochales-Palau, R.; Moens, M.-F.; and Milward, D. 2010. Approaches to text mining arguments from legal cases. In Semantic processing of legal texts. 60-79.

Yang, Z.; Yang, D.; Dyer, C.; He, X.; Smola, A.; and Hovy, E. 2016. Hierarchical attention networks for document classification. In NAACL 2016, 1480-1489.

Zhu, X., and Ghahramani, Z. 2002. Learning from labeled and unlabeled data with label propagation.

Zhu, J., and Hovy, E. 2007. Active learning for word sense disambiguation with methods for addressing the class imbalance problem. In EMNLP 2007, 783-790. 